\title{
ORIGINE DES RÉGIONS ACTIVES SOLAIRES 'ANORMALES'
}

\author{
M. J. Martres \\ (Observatoire de Meudon, S. \& O., France)
}

\begin{abstract}
A BSTRACT
Solar active regions are considered 'anomalous' when they belong to magnetic classes $\gamma, \beta \gamma$ and $\beta \mathrm{f}-\alpha \mathrm{f}$. The study of the solar activity of the region where, later on, these groups are born shows an evident correlation between the presence of an old active center and the complexity of the new active region.

It is found that the complexity is greater if the old active center is younger, and the superposition better. We also observe that the birth of anomalous sunspots groups occurs much more frequently on the western side of the magnetic inversion line of the old center.

When the birth of an active center occurs outside and on the West of the faculae, we observe the weakly anomalous groups $\beta \mathrm{f}-\alpha \mathrm{f}$. The 'perturbation' decreases with distance and is extended at least to 10 heliographic degrees of the boundaries of the old faculae.
\end{abstract}

La classe des groupes de taches $\beta \mathrm{p}-\alpha \mathrm{p}$ de la classification magnétique du MountWilson réunit environ $80 \%$ de l'ensemble des groupes de taches qui apparaissent sur le soleil, et il est facile de penser que les groupes ainsi définis correspondent à un type 'normal' de centre actif solaire.

Dans un ordre croissant de malformations, nous trouvons les $\beta \mathrm{f}-\alpha \mathrm{f}$, les $\beta \gamma$ et les $\gamma$. Par opposition nous pouvons donc penser que ce sont là des centres d'activité de type 'anormal'.

Utilisant d'une part les publications des données magnétiques et le film des observations journalières de Mount-Wilson, d'autre part la collection des spectrohéliogrammes de Meudon, nous avons cherché à associer la formation des centres actifs appartenant aux différentes classes magnétiques, à l'activité solaire préexistante de la région dans laquelle ils apparaissent. Cette étude est parfois gênée par les lacunes d'observations de l'un et l'autre des deux observatoires.

1. Au cours de la période 1920-35 nous avons revelé 307 groupes de taches d'importance supérieure à 5 dans l'échelle à 10 échelons des Cartes Synoptiques de la Chromosphère Solaire et de classe magnétique quelconque.

Ces groupes se trouvent répartis comme suit:

49 ont été signalés $\gamma$ au moins 1 jour,

119 ont été signalés $\beta \gamma$ au moins 1 jour,

139 n'ont jamais été classés $\gamma$ ou $\beta \gamma$.

Certains de ces groupes sont nés dans des régions solaires où aucune trace faculaire

Kiepenheuer (ed.). Structure and Development of Solar Active Regions, 25-32. C I.A.U. 
n'est observable sur les clichés $K_{1}$, d'autres à l'emplacement ou très près de régions actives préexistantes.

Leur répartition en fonction de leur classe magnétique est indiquée dans l'histogramme de la Figure 1.

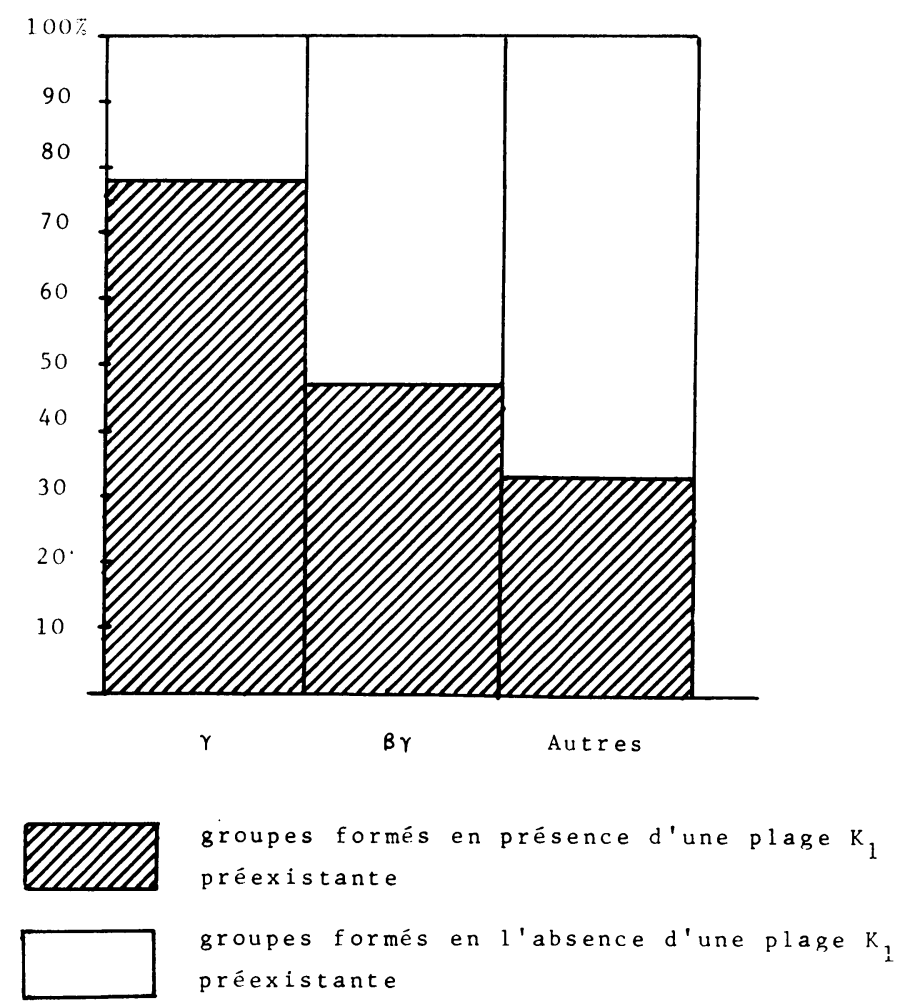

FIG. 1. Histogramme de la distribution des groupes en fonction de leur classe magnétique et de l'activité de la région où ils apparaissent.

Les pourcentages, malgré la sévérité des critères (complexité éphémère) et les lacunes des observations (un groupe peut naître et mourir pendant les 13 jours de passage d'une région dans l'hémisphère invisible du Soleil) semblent indiquer que la présence de C.A. préexistant influe sur la complexité des structures nouvelles.

2. Nous avons cherché à préciser certaines lois de l'interaction entre C.A. nouveau et C.A. ancien.

Beaucoup de variables entrent en jeu. Parmi celles-ci on trouve, d'une part, le degré de complexité du C.A. nouveau, d'autre part les importances respectives des deux centres actifs en présence, l'âge ou le stade de l'évolution du C.A. préexistant au moment de la naissance du second et aussi leur disposition respective. 
Nous nous sommes bornés à étudier pour les groupes de l'échantillon précédent nés dans des régions où l'existence d'un centre actif était observable, la corrélation avec la complexité magnétique:

(1) de l'âge de la formation préexistante: à savoir si celle-ci possède ou ne possède plus de taches visibles sur $\mathrm{K}_{1 \mathrm{v}}$;

(2) de leurs distances respectives: à savoir s'il y a superposition de leurs facules ou seulement juxtaposition.
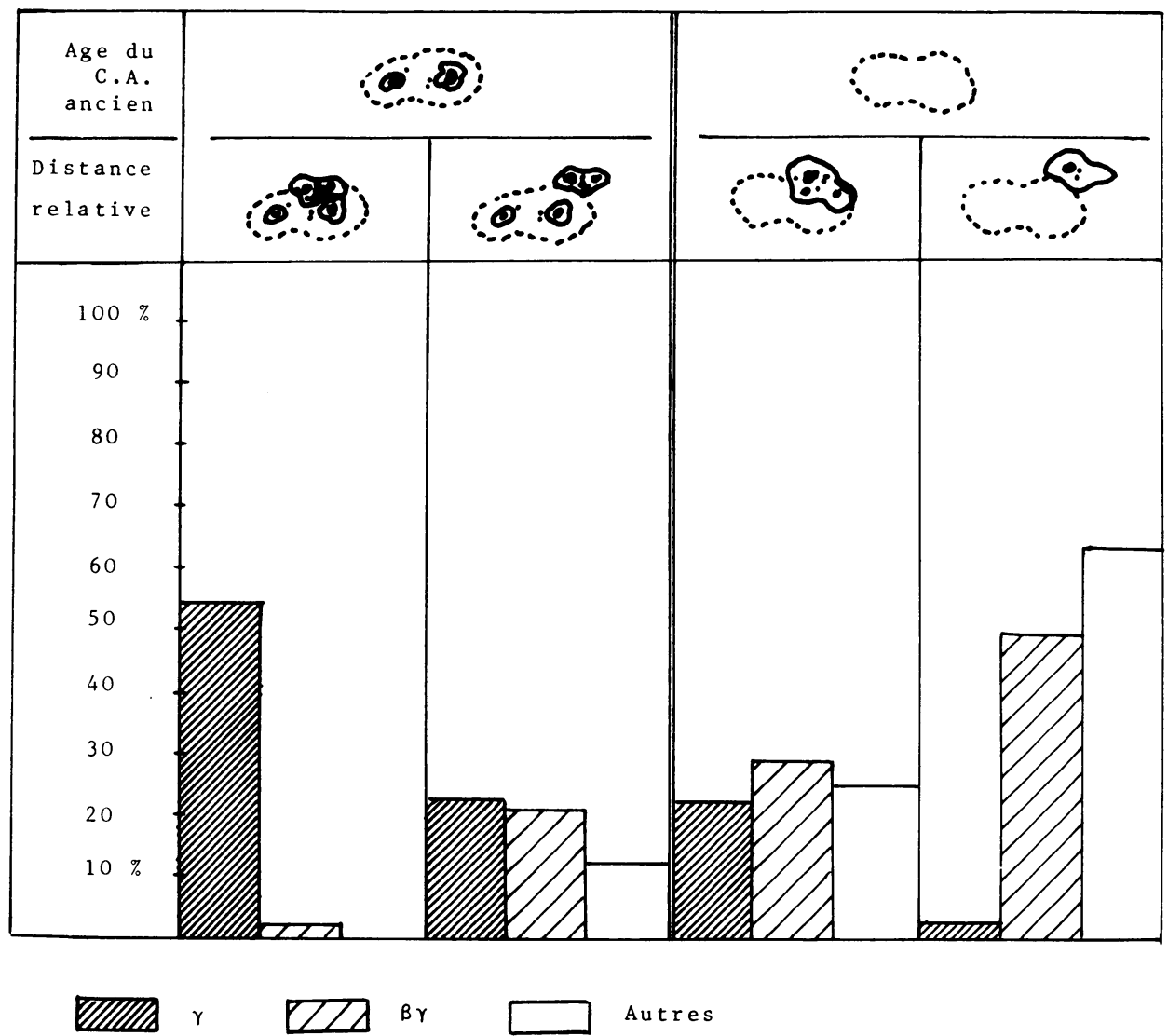

FIG. 2. Distribution des C.A. formés en présence d'un C.A. ancien en fonction de leur classe magnétique, de l'âge du C.A. ancien et de la distance relative.

Comme il est naturel on trouve (Figure 2) que la perturbation est d'autant plus forte que le C.A. ancien a des champs magnétiques plus forts (groupe jeune, encore taché) et que la superposition des deux formations est plus complète. 
3. Influence de la disposition relative.

Nous avons recherché si le fait qu'un C.A. nouveau se forme à l'Est ou à l'Ouest d'une formation préexistante joue un rôle dans la complexité magnétique et l'évolution du C.A. résultant.

Les points d'apparition des groupes $\gamma$ et $\beta \gamma$ (160 cas) ont été comparés à une droite fictive représentant grossièrement la ligne d'inversion magnétique du C.A. ancien.

On reconnaît que plus de $70 \%$ de ces centres complexes apparaissent à l'ouest de cette droite (quelques uns sur cette droite). En revanche, d'une manière générale, tous les groupes apparus à l'est comptent parmi les moins perturbés.

4. Cas des groupes faiblement anormaux $(\beta \mathrm{f}-\alpha \mathrm{f})$.

Etant donné le grand nombre des groupes simples nous avons réduit notre étude à tous les groupes classés $\beta \mathrm{p}-\alpha \mathrm{p}$ et $\beta \mathrm{f}-\alpha \mathrm{f}$ par les observateurs du Mount-Wilson pendant les années 1936-46 et 1955 (2è semestre) - 1956 (1er semestre).

Notre échantillon se trouve composé de 733 cas, répartis en $580 \beta \mathrm{p}$ - $x \mathrm{p}$, et $153 \beta \mathrm{f}-\alpha \mathrm{f}$.

Nous constatons que dans notre échantillon tous les $\beta f-\alpha f$ se trouvent disposés à

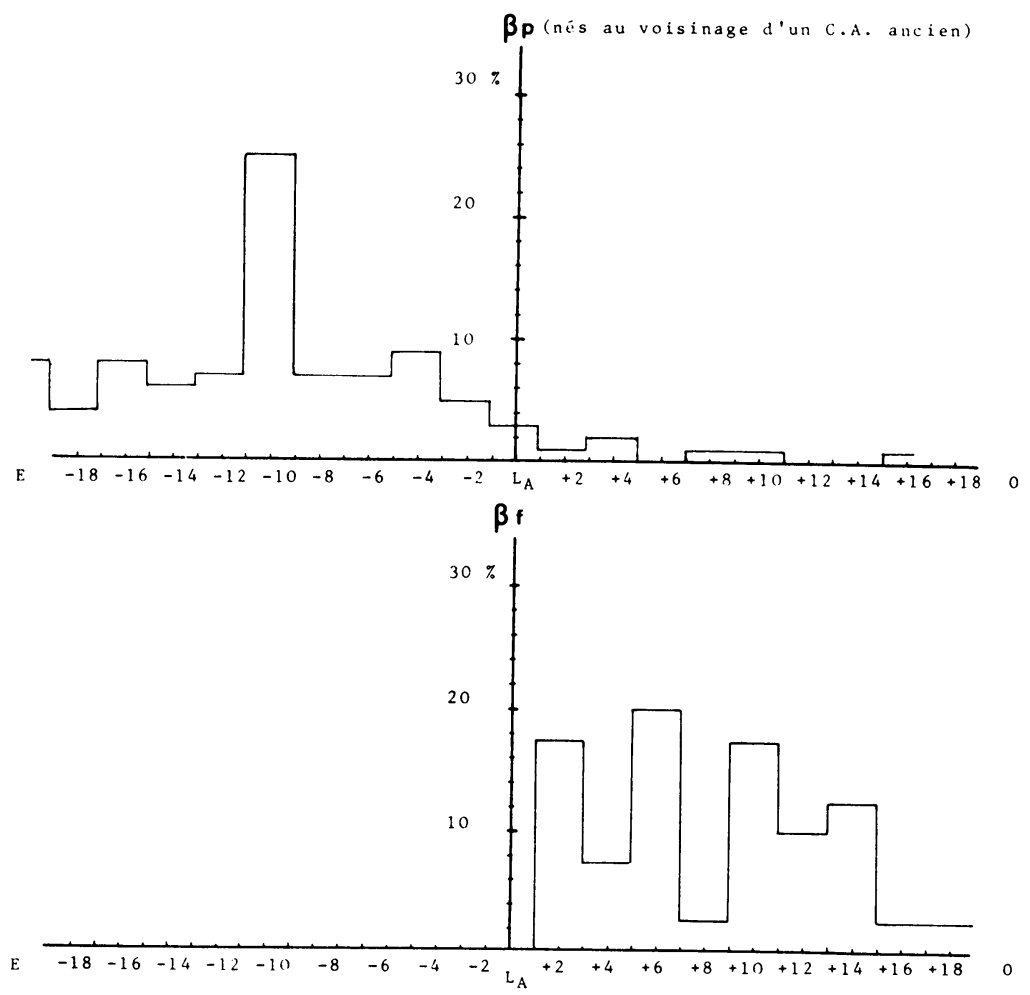

FIG. 3. Répartition des écarts en longitude $\Delta L . \Delta L=L_{N}-L_{A}$ des centres de figure des C.A.nouveau et ancien. 
l'ouest d'une R.A.préexistante* et en général à une distance égale ou inférieure à $10^{\circ}$ $\mathrm{du}$ bord de la facule de l'ancienne formation alors que les $\beta p$ - $\alpha p$ apparaissent soit dans des régions dépourvus de C.A. soit à l'est d'une région active préexistante (quelques cas sont juxtaposés mais en pile ordonnée avec celle-ci) (Figure 3).

La Figure 4 schématise l'ensemble des résultats.

\section{Est}

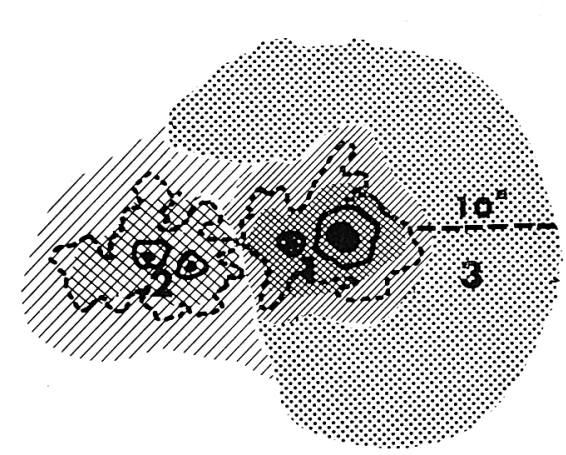

Ouest
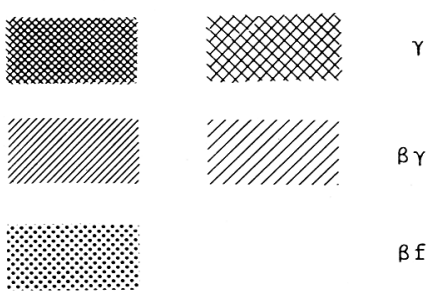

B $\gamma$

$\beta \mathrm{f}$

Fig. 4. Schéma d'un C.A. de l'hémisphère N. et disposition des aires où peuvent se développer les groupes anormaux.

Autour d'un centre actif solaire taché, supposé unique sur le Soleil, nous avons délimité les zones favorables à l'apparition des groupes anormaux.

Sachant que

$72 \%$ des groupes $\gamma$ apparaissent dans la zone 1 ,

$23 \%$ des groupes $\gamma$ apparaissent dans la zone 2 ,

$72 \%$ des groupes $\beta \gamma$ apparaissent dans la région limitrophe des zones $1-3$,

$21 \%$ des groupes $\beta \gamma$ apparaissent dans la région limitrophe des zones $2-4$,

$90 \%$ des groupes $\beta \mathrm{f}$ apparaissent dans la zone 3,

nous pouvons délimiter ainsi une aire grossièrement centrée sur la tache de tête du C.A. existant où, une naissance de C.A. venant à se produire on aura toutes les chances de voir se développer des groupes anormaux, d'autant plus perturbés que le centre préexistant sera jeune et qu'ils seront situés près de cette tache de tête.

* Ont été retirés de l'échantillon les groupes situés à moins de $10^{\circ}$ de plusieurs C.A. anciens. 

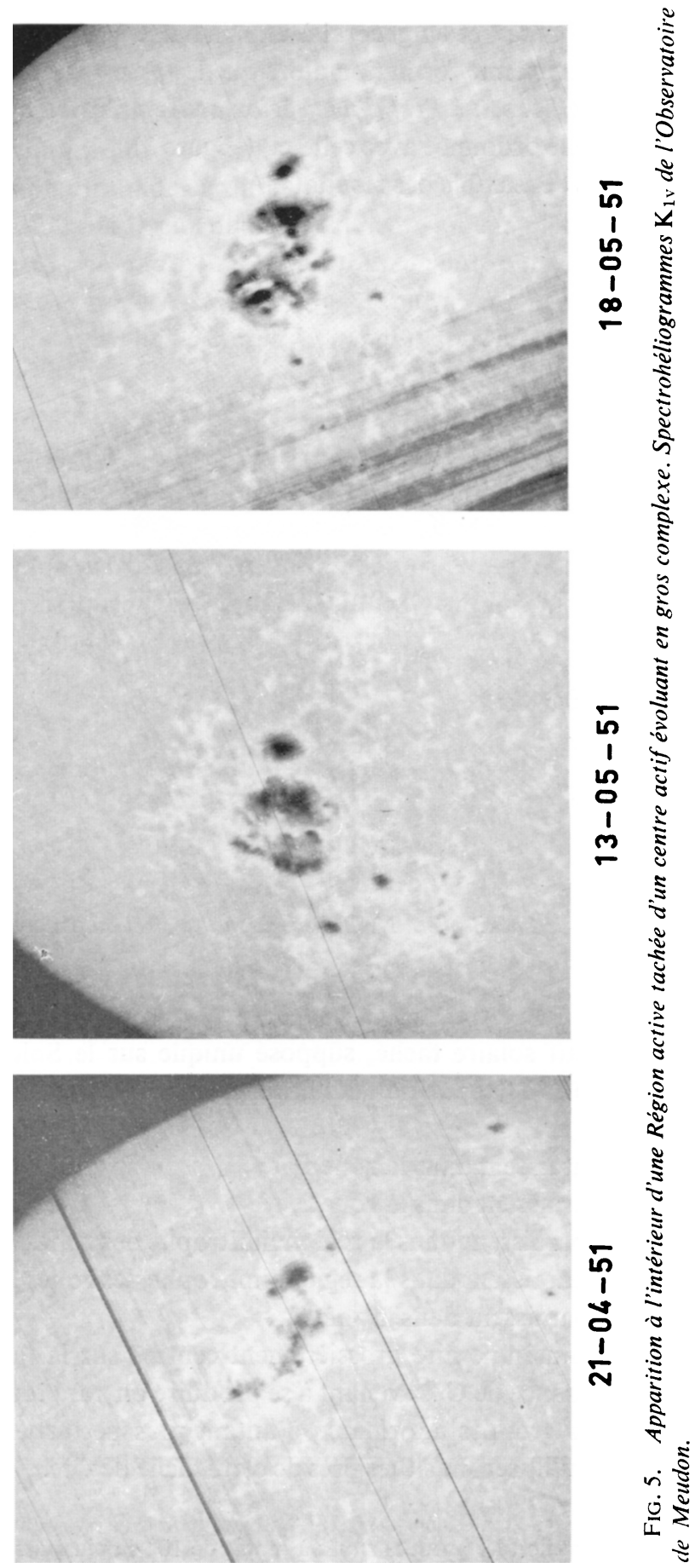
ORIGINE DES RÉGIONS ACTIVES SOLAIRES 'ANORMALES'
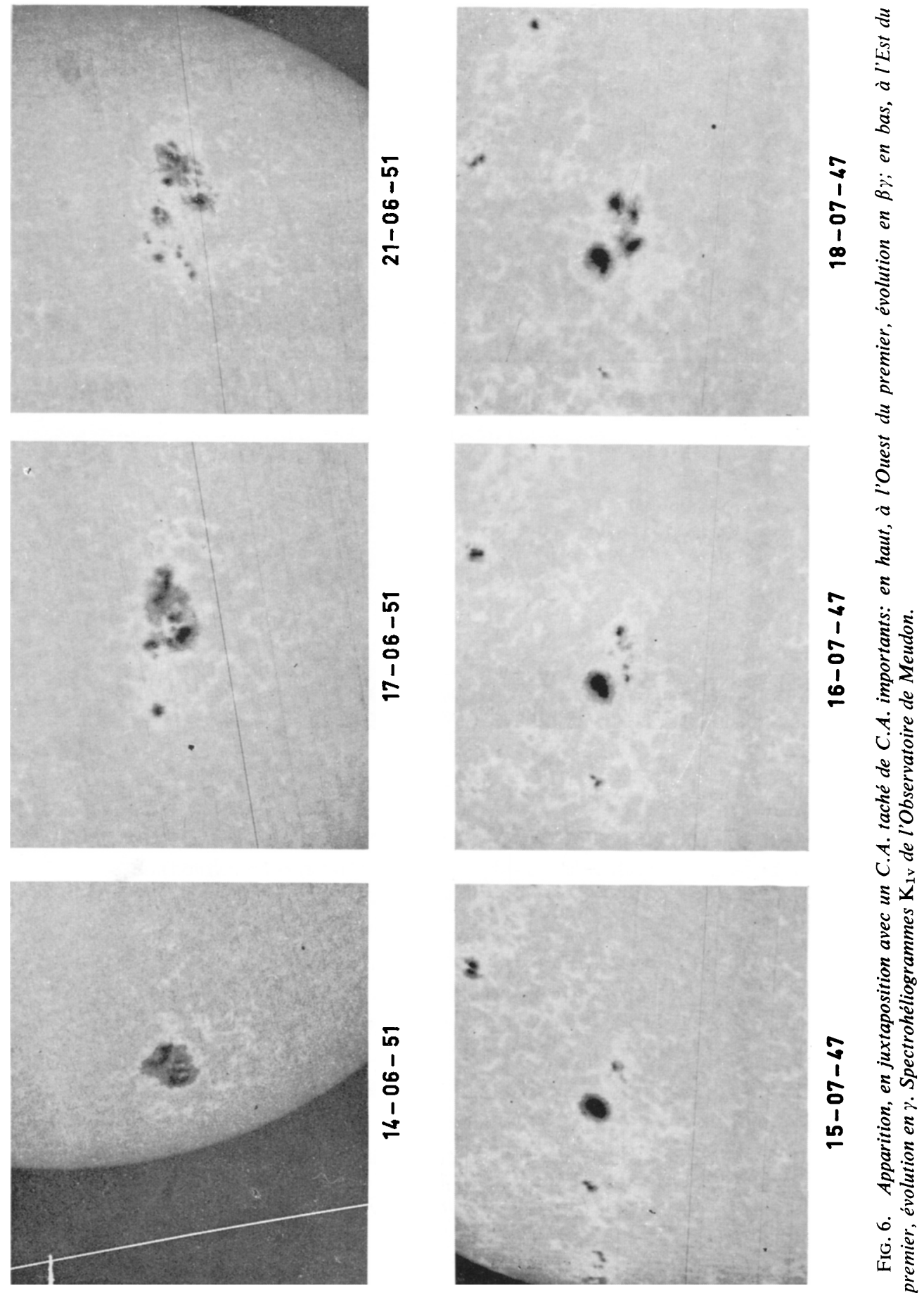


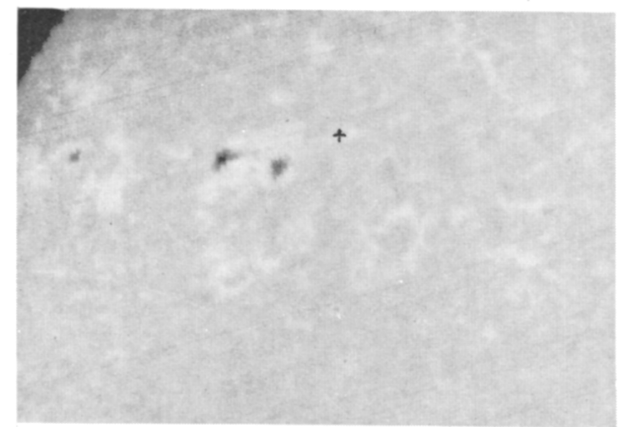

$23-01-37$

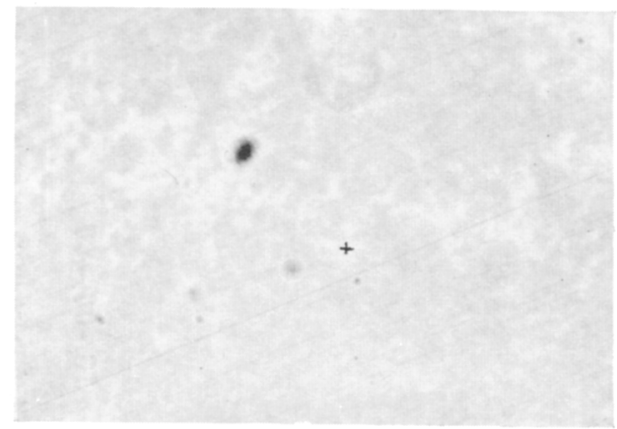

$07-05-58$

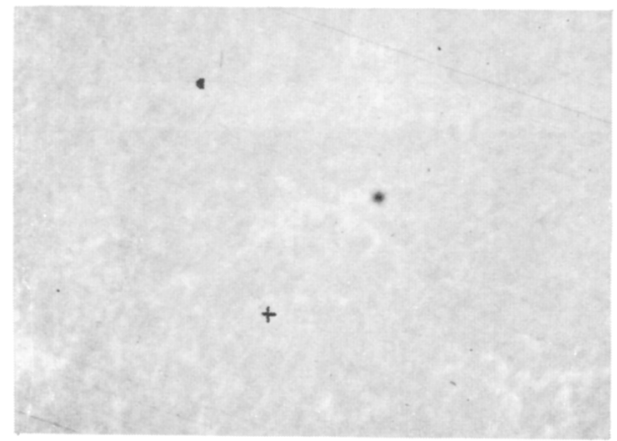

25-08-36
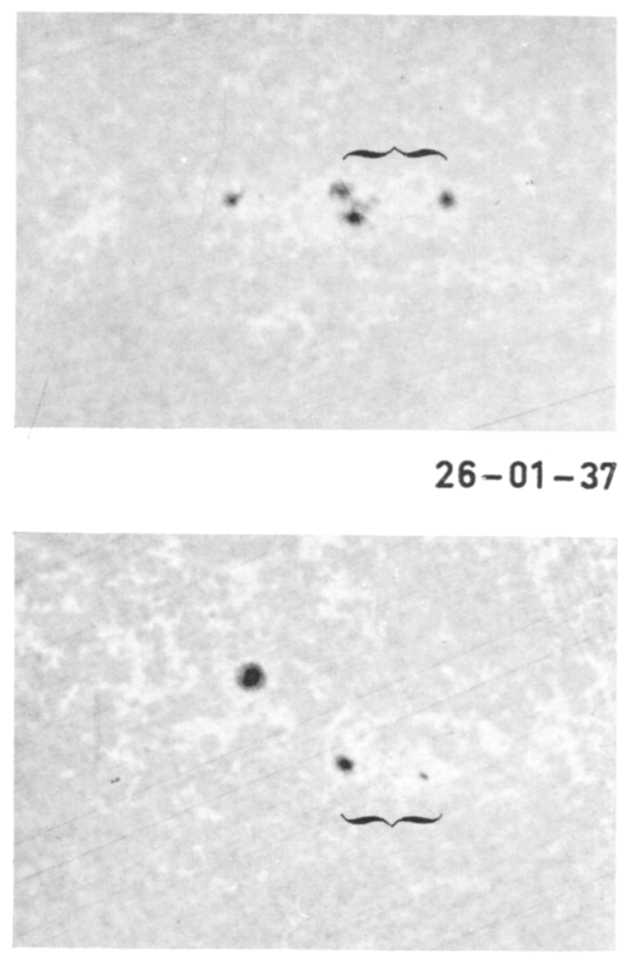

$09-05-58$

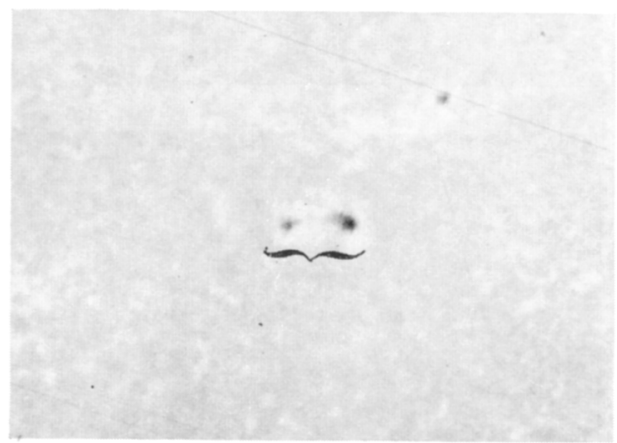

$27-08-36$

Fig. 7. Apparition de C.A. simples à moins de $10^{\circ}$ du centre préexistant. (1) en haut, centres jointifs. Formation nouvelle à l'Ouest, évolue en $\beta f$. (2) au centre, centres non jointifs. Formation nouvelle à l'Ouest, évolue en $\beta f$. (3) en bas, centres non jointifs. Formation nouvelle à l'Est, évolue en $\beta p$. Spectrohéliogrammes de l'Observatoire de Meudon. 